\title{
O gênero Gomphonema (Bacillariophyta) na comunidade perifítica do rio e reservatório de Curuá-Una (Santarém, Pará, Brasil)
}

The genus Gomphonema (Bacillariophyta) in the periphytic community of the Curuá-Una river and reservoir (Santarém, Pará, Brazil)

\author{
Jéssica da Silva Azevedo ${ }^{1,5}$, Dávia Marciana Talgatti ${ }^{1,2,5}$, Lezilda Carvalho Torgan ${ }^{3}$, \\ Andreia Cavalcante Pereira ${ }^{4} \&$ Sérgio de Melo $^{1,4}$
}

\begin{abstract}
Resumo
O gênero Gomphonema é bem representado em termos de riqueza e abundância em comunidades perifíticas nos ambientes aquáticos dulcícolas. Este estudo teve como objetivo conhecer a composição e riqueza do gênero Gomphonema aderido a macrófitas aquáticas na região litorânea do rio e reservatório de Curuá-Una. As amostragens foram realizadas em novembro/2011 (período seco) e julho/2013 (período chuvoso). Para a análise morfológica e morfométrica das diatomáceas as amostras foram oxidadas e preparadas em lâminas permanentes. O material foi estudado em microscopia ópticae eletrônica de varredura. Como resultados foram registrados 13 táxons específicos e infraespecíficos. Os mais representativos nos dois períodos hidrológicos foram: G. parvulum var. lagenula, G. naviculoides e G. pantropicum, sendo o primeiro amplamente citado na flora brasileira, e o último considerado primeiro registro para o Brasil e Pará.
\end{abstract}

Palavras-chaves: Amazônia, diatomáceas, perifiton, taxonomia.

\begin{abstract}
The Gomphonema genus is well represented in terms of richness and abundance in periphytic communities in freshwater environments. This study aimed to know the composition and richness of the Gomphonema genus attached to aquatic macrophytes in the littoral zone of the Curuá-Una river and reservoir. Samplings were performed in November/2011 (dry period) and July/2013 (rainy period). For the morphological and morphometric analysis of the diatoms the samples were oxidized and prepared in permanent slides. The material was studied under optical and scanning electron microscopy. As results we registered 13 specific and infraspecific Gomphonema taxa. The most representative in the two hydrological periods were: G. parvulum var. lagenula, G. pantropicum, and G. naviculoides, the first are widely cited in Brazilian flora, and the last considered first record to Brazil and Pará.
\end{abstract}

Key words: Amazon, diatoms, periphyton, taxonomy.

\section{Introdução}

Os representantes de Gomphonemataceae Kützing são registrados principalmente em ambientes dulcícolas e se destacam em riqueza de espécies e abundância de indivíduos nas comunidades perifíticas (Round et al.1990). Esta família está constituída atualmente por quatro gêneros: Gomphonema Ehrenberg, Gomphoneis Cleve, Gomphosinica Kociolek et al. e Gomphocymbella Otto Müller (Kociolek 2016).
Gomphonema é bastante comum em comunidades haptobentônicas de água doce, principalmente no perifíton, por formar pedúnculo de mucilagem através do campo de poros localizados no polo basal da valva, facilitando a adesão a substratos sólidos. Este gênero é caracterizado por apresentar células heteropolares em vista valvar e cuneiformes em vista lateral. As valvas podem ser lineares a lanceoladas, com polo basal estreito e polo apical mais largo, que variam de rostrados a

\footnotetext{
${ }^{1}$ Universidade Federal do Oeste do Pará, PPG Sociedade, Natureza e Desenvolvimento, Av. Mendonça Furtado 2440, Bairro Aparecida, 6840-070, Santarém, PA, Brasil.

${ }^{2}$ Universidade Federal do Oeste do Pará, Campus Oriximiná, Rod. PA-254, 257, Bairro Santíssimo, 68270-000, Oriximiná, PA, Brasil. daviatalgatti@gmail.com

${ }^{3}$ Fundação Zoobotânica do Rio Grande do Sul, Museus de Ciências Naturais, R. Salvador França 1427, Bairro Jardim Botânico, 90690-000, Porto Alegre, RS, Brasil.

${ }^{4}$ Universidade Federal do Oeste do Pará - Ufopa, Inst. Ciências e Tecnologia das Águas (ICTA), Av. Mendonça Furtado 2946, Bairro Fátima, 68040-050, Santarém, PA, Brasil.

${ }^{5}$ Autor para correspondência: jbioazevedo@gmail.com
} 
capitados. Possuem estrias unisseriadas, contendo poros geralmente ocluídos por uma vola reniforme única (Round et al. 1990). Entretanto por apresentar amplas variações morfológicas das frústulas, as características interespecíficas precisam ser cuidadosamente analisadas.

No Brasil, são escassos os estudos taxonômicos que abordam o gênero Gomphonema, entre estes os principais foram os de Schneck et al. (2008), Tremarin et al. (2009), Faria et al. (2010) e Bes et al. (2012). Na Amazônia as pesquisas realizadas com algas perifíticas são bastante incipientes (Putz \& Junk 1997; Castro 1999; Díaz-Castro et al. 2003; França et al. 2011; Wetzel et al. 2012a,b), além disso são poucos os estudos taxonômicos que envolvem o gênero Gomphonema. No estado do Pará, embora os estudos da diatomoflórula perifítica sejam raros, salientam-se os realizados por Burliga et al. (2007), Burliga \& Kociolek (2010, 2012), Bastos et al. (2011) e Tremarin et al. (2008, 2015). Em reservatórios as pesquisas são ainda mais raras limitando-se ao Reservatório de
Balbina no Amazonas realizados por BittencourtOliveira (1993a, 1993b, 1993c, 1994, 1995, 1997).

Com o intuito de ampliar o conhecimento da flora de Gomphonemataceae em regiões tropicais, o presente estudo teve como objetivo conhecer a composição e riqueza do gênero Gomphonema aderido a macrófitas aquáticas na região litorânea do rio e reservatório da Usina Hidrelétrica de Curuá-Una.

\section{Material e Métodos}

O reservatório de Curuá-Una (2²4'52"S $\left.-54^{\circ} 42^{\prime} 35^{\prime \prime} \mathrm{W}\right)$ dista 70 quilômetros do município de Santarém-PA, possui formato alongado e estreito, devido ao nível da água acompanhar o formato natural da bacia hidrográfica do rio Curuá- Una, e tem como principais afluentes os rios Moju, Mojuí e Igarapé Poraquê. Segundo Junk et al. (1981) e Vieira (2000) o rio Curuá-Una e seus afluentes são definidos como corpos d'águas claras.

As amostragens foram realizadas em período seco (novembro/2011) e chuvoso (julho/2013),

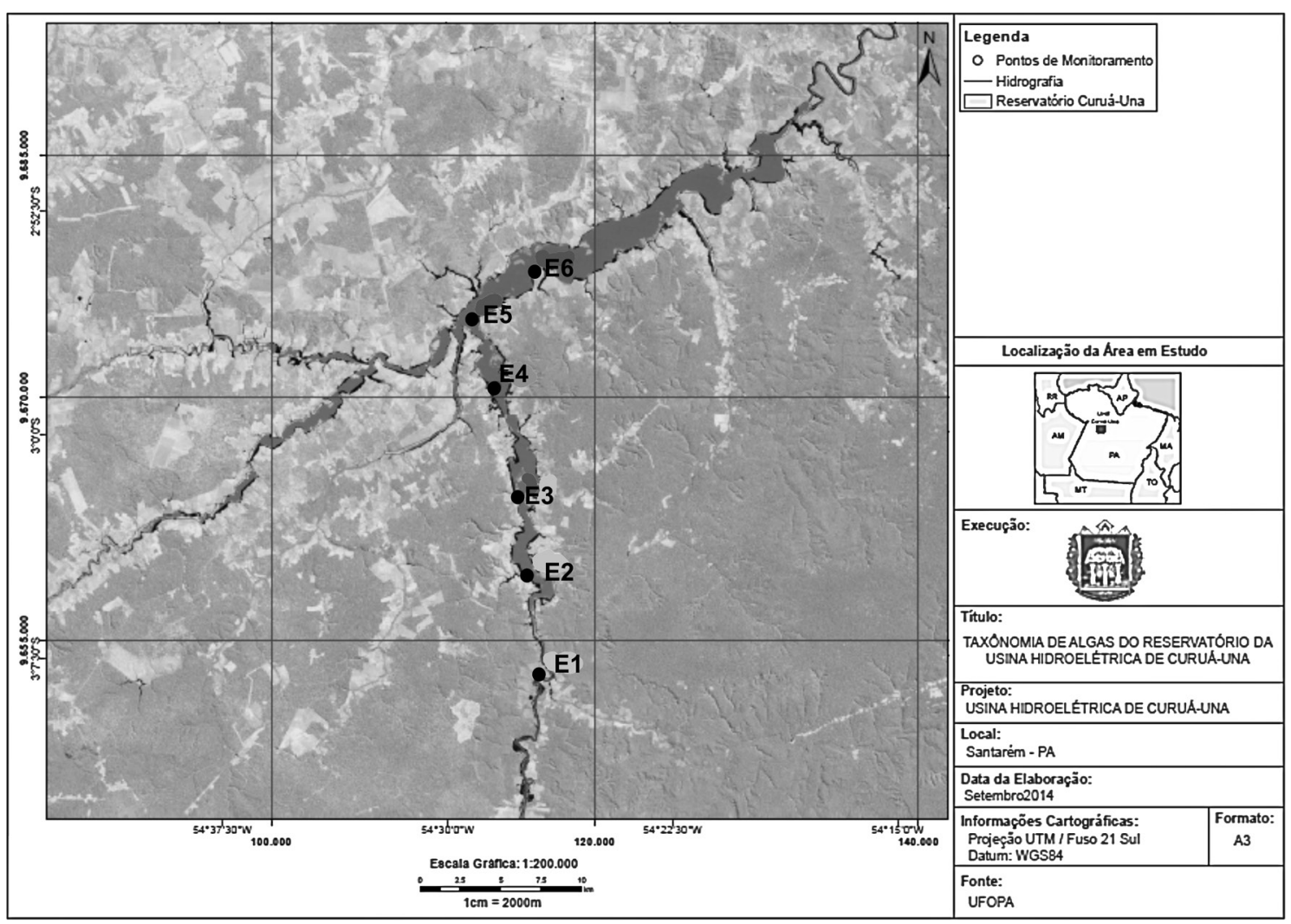

Figura 1 - Imagem georreferenciada do rio e reservatório de Curuá-Una, indicando as estações de amostragem E1E6. Fonte: Modificado da Universidade Federal do Oeste do Pará-UFOPA.

Figure 1 - Georeferenced image of the river and of Curuá-Una reservoir, indicating the E1-E6 sampling stations. Modified from Universidade Federal do Oeste do Pará-UFOPA. 
em seis estações de coleta (Fig.1), totalizando 12 amostras. O material foi obtido manualmente por meio da retirada de algumas porções vegetativas das macrófitas aquáticas mais representativas do local (Ludwigia sedoides (H.B.K) Hara e representantes de Poaceae). Posteriormente, foi efetuada a raspagem do substrato com escova de dente com auxílio de jatos de água destilada. As amostras foram preservadas com solução de formalina 4\% (Bicudo \& Menezes 2006) e depositadas no Herbário de Santarém (HSTM) da Universidade Federal do Oeste do Pará (Tab. 1).

Para o estudo da parede silicosa das diatomáceas, parte das amostras foi oxidada seguindo o método de Stoch (1970) e, após a lavagem, o material foi incluído entre lâmina e lamínula com resina Naphrax ${ }^{\circledR}$.

As análises foram realizadas ao microscópio óptico trinocular (modelo Axiostarplus-Carl Zeiss ${ }^{\circledR}$ ), as imagens foram obtidas com câmera de captura de imagens Zeiss (AxioCam ERc5s), posteriormente realizadas as medidas morfométricas de todos os táxons encontrados com o auxílio do programa Zen. Adicionalmente, alíquotas do material oxidado foram secas em suportes metálicos (stubs) e metalizados com uma camada de ouro paládio, no equipamento BAL-TEC SCb005. Após esta metalização, o material foi observado em microscópio eletrônico de varredura (MEV) JEOL 6060, operado com $10 \mathrm{~mm}$ de distância de trabalho e $15 \mathrm{kV}$.
A identificação das espécies de diatomáceas foi baseada em literatura especializada (Frenguelli 1923; Hustedt 1930, 1942; Cleve \& Euler 1955; Patrick \& Reimer 1975; Krammer \& Lange-Bertalot 1985, 1986, 1991; Lange-Bertalot 1995; Metzeltin \& Lange-Bertalot 1998, 2007; Metzeltin, LangeBertalot \& García-Rodríguez 2005; Sar et al. 2009; Reichardt 2015a,b). A classificação taxonômica adotada foi a de Round et al. (1990) e a terminologia utilizada nas descrições dos táxons foi baseada em Hendey (1964) e Barber \& Haworth (1981).

\section{Resultados e Discussão}

O estudo revelou a presença de 13 táxons de Gomphonema, sendo dez identificados em nível específico, um em nível de variedade e dois apenas em nível genérico, destes oito constituem primeiras citações para o estado do Pará. Os táxons estão descritos e ilustrados a seguir com informações morfológicas observadas em microscopia óptica e eletrônica de varredura.

1. Gomphonema archaevibrio Lange-Bertalot \& E. Reichardt in Lange-Bertalot. Icon. Diatom., v.1, p.32, pl. 26, figs. 8, 10-11, pl. 27, figs.1-7. 1995.

Figs. 2a,b; 3a-d

Valvas lanceoladas, polo apical arredondado levemente mais largo que o polo basal. Área axial estreita, linear. Rafe levemente sinuosa com extremidades proximais retas. Estrias paralelas

Tabela 1 - Dados de referências das amostras coletadas no rio e reservatório de Curuá-Una, indicando o número de registro no herbário, data de coleta, estações amostradas e coletores.

Table 1 - Reference data of the samples collected in the river and Curuá-Una reservoir, indicating the registration number in the herbarium, date of collection, stations and collectors.

\begin{tabular}{cccl}
\hline Material Examinado & Data & Estações & Coletores \\
\hline HSTM 000048 & $11 / 2011$ & 1 & S. Melo s.n. \\
HSTM 000049 & $11 / 2011$ & 2 & S. Melo s.n. \\
HSTM 000050 & $11 / 2011$ & 3 & S. Melo s.n \\
HSTM 000051 & $11 / 2011$ & 4 & S. Melo s.n \\
HSTM 000052 & $11 / 2011$ & 5 & S. Melo s.n. \\
HSTM 000053 & $11 / 2011$ & 6 & S. Melo s.n \\
HSTM 000054 & $11 / 07 / 2013$ & 1 & Azevedo, J.S. s.n. \& Melo, S.; Burliga, A.L. \\
HSTM 000055 & $11 / 07 / 2013$ & 2 & Azevedo, J.S. s.n. \& Melo, S.; Burliga, A.L. \\
HSTM 000056 & $11 / 07 / 2013$ & 3 & Azevedo, J.S. s.n. \& Melo, S.; Burliga, A.L. \\
HSTM 000057 & $11 / 07 / 2013$ & 4 & Azevedo, J.S. s.n. \& Melo, S.; Burliga, A.L. \\
HSTM 000058 & $11 / 07 / 2013$ & 5 & Azevedo, J.S. s.n. \& Melo, S.; Burliga, A.L. \\
HSTM 000059 & $11 / 07 / 2013$ & 6 & Azevedo, J.S. s.n. \& Melo, S.; Burliga, A.L. \\
\hline
\end{tabular}



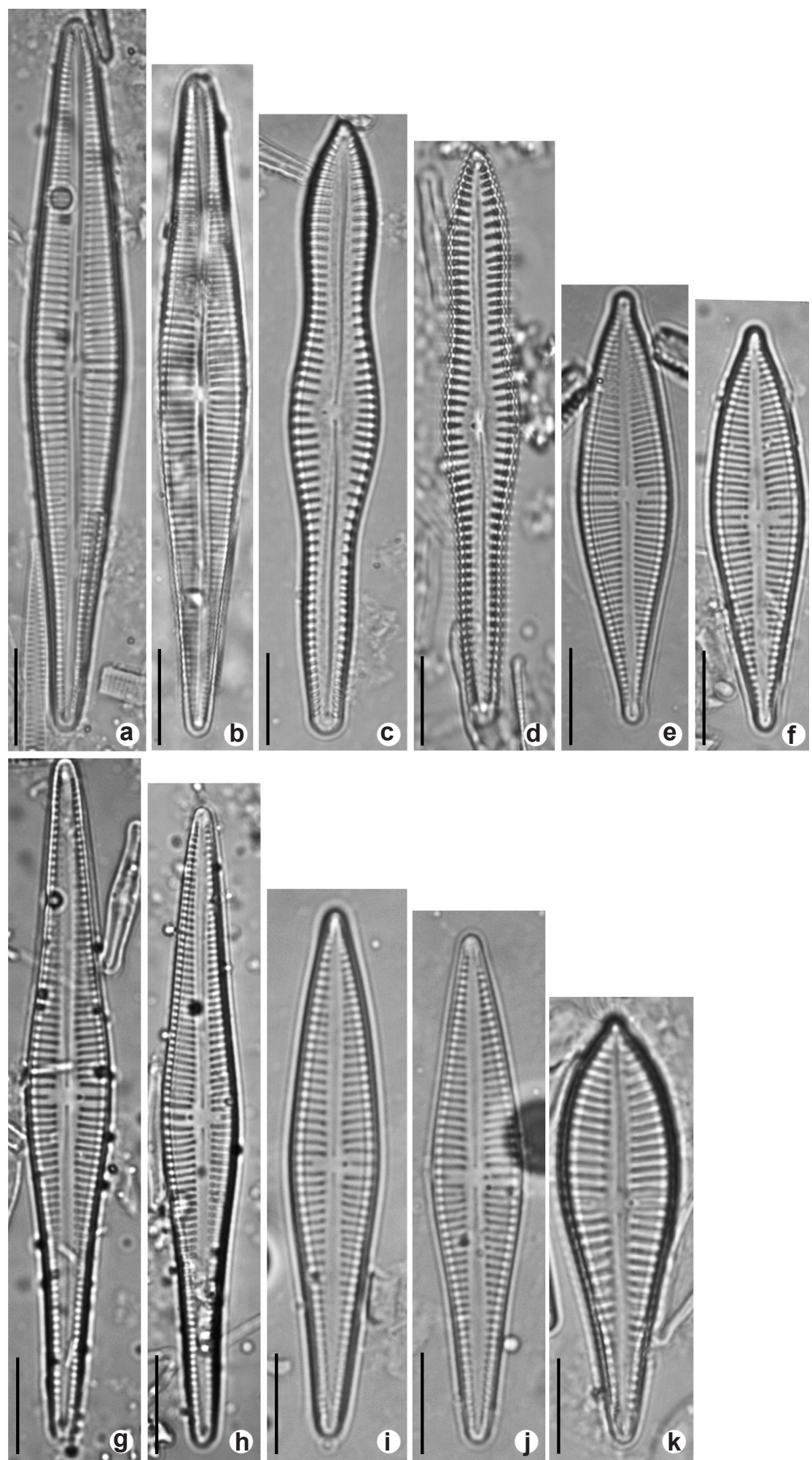

Figura 2 - Imagens em microscopia óptica - a,b. G. archaevibrio; c,d. G. butantanum; e,f. G. grunowii; g,h. G. guaraniarum; i,j. G. naviculoides; k. G. neoapiculatum. Escala $=10 \mu \mathrm{m}$.

Figure 2 - Images in light microscopy - a,b. G. archaevibrio; c,d. G. butantanum; e,f. G. grunowii; g,h. G. guaraniarum; i,j. G. naviculoides; k. G. neoapiculatum. Scale $=10 \mu \mathrm{m}$. 
a levemente radiadas. Estigma presente junto à estria mediana encurtada. Em MEV, vista interna, observa-se estigma em forma de fenda transversal e aréolas arredondadas (Fig. 3c). Presença de campo de poros no polo basal, vista interna (Fig. 3d). Comprimento 63,63-109,6 $\mu \mathrm{m}$, largura 9,5-12,4 $\mu \mathrm{m}$, estrias 9-12 em $10 \mu \mathrm{m}$ na área central, $12-13$ estrias em $10 \mu \mathrm{m}$ nos polos.

Material examinado: HSTM 000053 , HSTM 000054. Comentário: os indivíduos $(\mathrm{n}=7)$ assemelham-se ao tipo da "Lagune des Amazonas" Santarém, Pará (Reichardt 1995) e às populações encontradas em outros ambientes da Região Amazônica, como bacia do Rio Negro (Wetzel 2011), bacia do Rio Amazonas (Metzeltin \& Lange-Bertalot 1998) e Rio Tapajós (Metzeltin \& Lange Bertalot 2007). Entretanto, os indivíduos estudados apresentaram valvas menores ao observado no material tipo.

2. Gomphonema butantanum Krasske, Svensk Botanisk Tidsrift, v. 42, p. 443, pl. 2, figs.1819.1948.

Fig. 2c,d

Valvas clavadas com margens onduladas, polo apical cuneado a sub-rostrado, polo basal atenuado arredondado. Área axial alargando-se em direção à área central. Rafe linear a levemente onduladas com extremidades proximais retas. Estrias unisseriadas curtas e grosseiras levemente radiadas, com aréolas em fenda longitudinal. Estigma presente próximo às extremidades proximais da rafe. Comprimento 47-77,7 $\mu \mathrm{m}$, largura 7,8-15,2 $\mu \mathrm{m}$, estrias $8-9 \mathrm{em}$ $10 \mu \mathrm{m}$ na área central, $10-11$ estrias em $10 \mu \mathrm{m}$ nos polos.

Material examinado: HSTM 000051 , HSTM 000054. Comentário: os indivíduos $(\mathrm{n}=3)$ observados apresentam morfologia semelhante e com menor comprimento aos indivíduos encontrados por Krasske $(1948$, p. 437, figs. 18, 19) aderido à espécie Lophocolea aquatica Herzog, em Butantan, São Paulo, Brasil. Esta espécie pode ser comparada com a Gomphonema longiceps f. suecica Grunow (Hustedt 1930, p. 375, fig. 708) diferenciando-se por apresentar maior comprimento e densidade de estrias (10-12 em $10 \mu \mathrm{m})$. Esta espécie é primeira citação para o estado do Pará.

3. Gomphonema grunowii R.M. Patrick \& Reimer in Acad. Nat. Sci. Phil., Monog. v.2, p. 131, pl. 17, fig. 6. 1975.

Fig. 2e,f

Valvas lanceoladas, polo apical atenuado subrostrado, polo basal atenuado arredondado. Área axial estreita, linear. Rafe linear com extremidades proximais retas. Estrias radiadas. Estigma presente no lado oposto da estria mediana encurtada. Aréolas arredondas. Comprimento 30-35,8 $\mu \mathrm{m}$, largura $7-9,2 \mu \mathrm{m}$, estrias $12-14$ em $10 \mu \mathrm{m}$ na área central e 15 estrias nos polos.

Material examinado: HSTM 000051, HSTM 000054, HSTM 000055.

Comentário: os indivíduos $(\mathrm{n}=13)$ observados assemelham-se morfologicamente aos espécimes apresentados por Patrick \& Reimer (1975, plate 159, figs.1-4) originários da Guiana Francesa e conferem quanto às dimensões (compr. 33-56 $\mu \mathrm{m}$, larg.7-10 $\mu \mathrm{m}$ e estrias 12-13 em $10 \mu \mathrm{m})$. Esta espécie é primeira citação para o estado do Pará.

4. Gomphonema guaraniarum Metzeltin \& Lange- Bertalot in Lange-Bertalot. Icon. Diatom., v.18, p. 147, pl. 212, figs.9-14. 2007 . Fig. 2g,h

Valvas lanceoladas, polo apical agudo, polo basal semelhante ao apical. Área axial estreita, linear. Rafe levemente sinuosa com extremidades proximais retas. Estrias radiadas, levemente mais distantes entre si na área central, algumas vezes paralelas nos polos. Estigma presente junto à estria mediana encurtada. Comprimento 50-106,4 $\mu \mathrm{m}$, largura 9,5-12,4 $\mu \mathrm{m}$, estrias 11-14 em $10 \mu \mathrm{m}$ na área central, 13-14 estrias em $10 \mu \mathrm{m}$ nos polos. Material examinado: HSTM 000049, HSTM 000050, HSTM 000051, HSTM 000054, HSTM 000056, HSTM 000057 , HSTM 000059.

Comentário: a população $(n=19)$ observada assemelha-se morfologicamente ao material tipo apresentado por Metzeltin \& Lange- Bertalot (2007, pág.694, figs.9-14), no Arroyo Hondo, pantanal do Paraguay. Observou-se nesta população que os indivíduos apresentaram semelhanças nas dimensões e densidade de estrias (compr. 50-90 $\mu \mathrm{m}$, larg. 10-12 $\mu \mathrm{m}$, estrias 10-12 em $10 \mu \mathrm{m}$ ). No material tipo, como no identificado por Wetzel (2011) foram observados espécimes abaixo de $65,7 \mu \mathrm{m}$ de comprimento. Esta espécie é primeira citação para o estado do Pará.

5. Gomphonema naviculoides W. Smith in Syn. Brit. Diat. v.2, p. 107, pl. 32-60, 61-62.1856.

Figs. 2i,j; 3e,f

Valvas lanceoladas a rombo-lanceoladas, polo apical atenuado, polo basal atenuado arredondado. Área axial linear alargando-se em direção à área central. Rafe linear com extremidades proximais retas. Estrias paralelas a levemente radiadas. Estigma presente no lado oposto da estria mediana encurtada. Em MEV, vista externa, estigma em forma arredondada, situado ao lado oposto da 


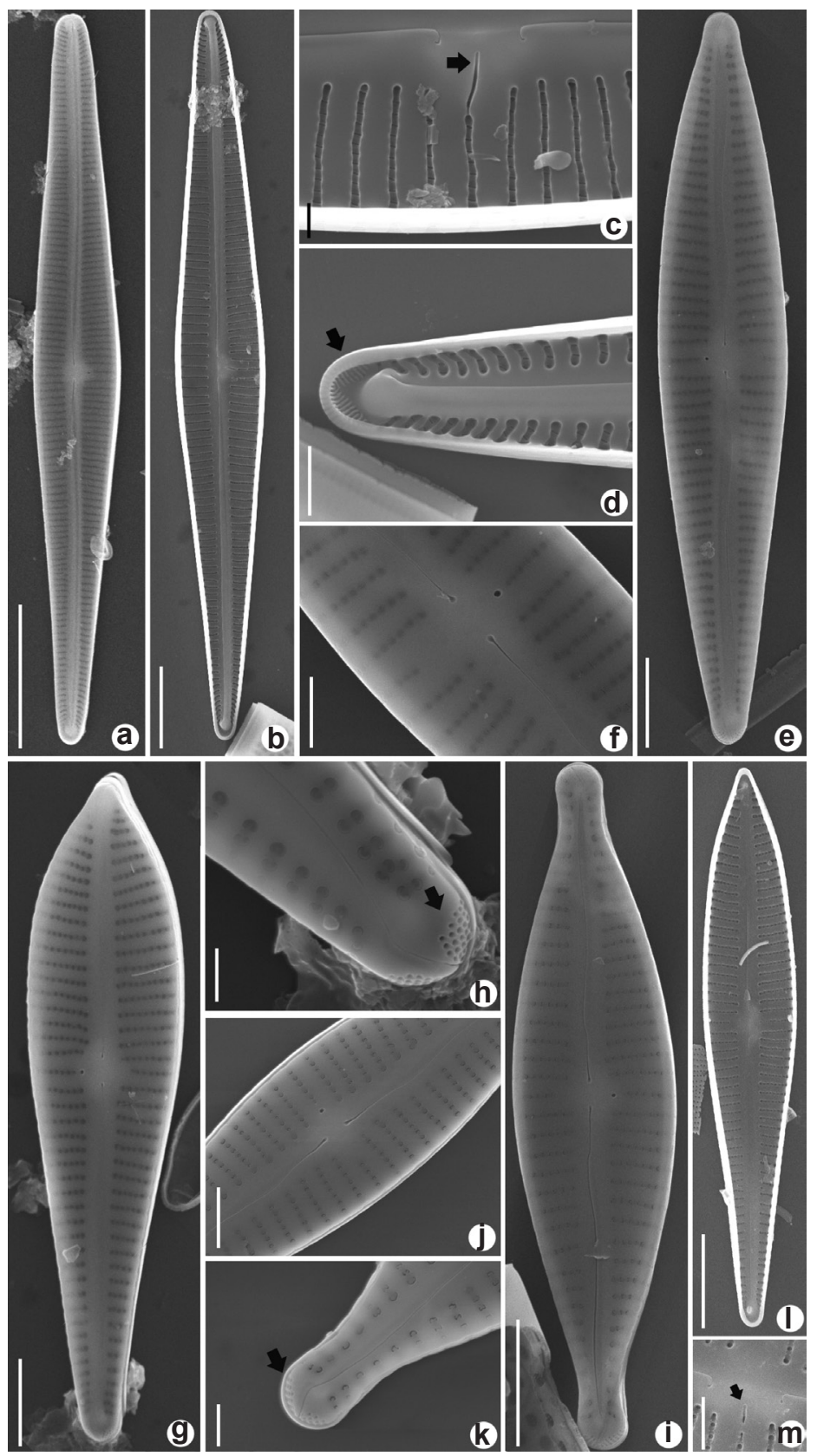

Figura 3 - Imagens em microscopia eletrônica de varredura - a-d. G. archaevibrio - a. vista geral externa da valva; b. vista geral interna da valva; c. vista interna das extremidades proximais da rafe e seta indicando a abertura do estigma; $d$. seta indicando campo de poros no polo basal da valva; e,f. G. naviculoides - e. vista geral externa da valva; f. vista externa das extremidades proximais da rafe na área central; g,h. G. neoapiculatum - g. vista geral externa da valva; h. seta indicando campo de poros no polo basal da valva; i-k. G. parvulum var. lagenula - i. vista geral externa da valva; j. vista externa das extremidades proximais da rafe na área central e abertura do estigma; $\mathrm{k}$. seta indicando campo de poros; $1, \mathrm{~m}$. G. perapicatum - 1. vista geral interna da valva; $\mathrm{m}$. detalhe da região central mostrando o estigma (seta). Escalas: a. $20 \mu \mathrm{m}$, b,l. $10 \mu \mathrm{m}$, e,g,i. $5 \mu \mathrm{m}$, d,f,j,m. $2 \mu \mathrm{m}, \mathrm{c}, \mathrm{h}, \mathrm{k} .1 \mu \mathrm{m}$.

Figure 3 - Images in scanning electron microscopy - a-d. G. archaevibrio - a. external general view of the valve; $b$. internal general view of the valve; c. internal view of valve showing of the central raphe ends and stigma opening (arrow); $d$. arrow indicating the pore field in the footpole of the valve; e,f. G. naviculoides - e. external view of the valve; f. external view of the central raphe fissures; g,h. G. neoapiculatum - g. external view of the valve; h. arrow indicating the pore field in the footpole of the valve; i-k. G. parvulum var. lagenula $-\mathrm{i}$. external view of the valve; j. external view of the central raphe fissures and opening of stigma; $\mathrm{k}$. Arrow indicating the pore field; $1-\mathrm{m}$. G. perapicatum -1 . internal view of the valve; $\mathrm{m}$. detail of the central of valve showing the stigma. Scales: a. $20 \mu \mathrm{m}, \mathrm{b}, 1.10 \mu \mathrm{m}$, e,g,i. $5 \mu \mathrm{m}$, d,f,j,m. $2 \mu \mathrm{m}$, c,h,k. $1 \mu \mathrm{m}$. 
estria mediana encurtada Aréolas unisseriadas arredondadas (5 aréolas em $2 \mu \mathrm{m}$ ) (Fig. 3e). Comprimento 19,7-45,88 $\mu \mathrm{m}$, largura 4,6-8,15 $\mu \mathrm{m}$, estrias $12-14$ em $10 \mu \mathrm{m}$ na área central e 12-16 estrias em $10 \mu \mathrm{m}$ nos polos.

Material examinado: HSTM 000049, HSTM 000050, HSTM 000051, HSTM 000054, HSTM 000055, HSTM 000056 , HSTM 000057, HSTM 000058, HSTM 000059.

Comentário: a identificação da população $(\mathrm{n}=$ 8) baseou-se no material descrito e ilustrado por Reichardt (2015, pág. 372, figs. 18-35, 62-63, compr. 35-45 $\mu \mathrm{m}$, larg. 7.8-9.5, estrias: 12-14 em $10 \mu \mathrm{m})$. Historicamente este táxon foi enquadrado em Gomphonema gracile Ehrenberg. No entanto, Reichardt (2015) revisou o material original de G. gracile observando diferenças taxonômicas importantes que justificaram o desmembramento do complexo em oito espécies, sendo uma delas G. naviculoides.

6. Gomphonema neoapiculatum Lange-Bertalot, E. Reichardt \& Metzeltin in Lange-Bertalot. Icon. Diatom., v.5, p. 120, pl. 157, figs 6-9. 1998.

Fig. 2k; 3g,h

Valvas clavadas, polo apical apiculado, polo basal atenuado arredondado. Área axial estreita, linear. Rafe levemente sinuosa com extremidades proximais retas. Estrias levemente radiadas. Estigma presente no lado oposto da estria mediana encurtada. Em MEV, vista externa, observa-se aréolas arredondadas (Fig. $3 \mathrm{~g}$ ) presença de campo de poros no polo basal, vista externa (Fig. 3h). Comprimento 34,5-39,4 $\mu \mathrm{m}$, largura 11,2-16 $\mu \mathrm{m}$, estrias $11 \mathrm{em} 10 \mu \mathrm{m}$ na área central, 11 estrias em $10 \mu \mathrm{m}$ nos polos.

Material examinado: HSTM 000051.

Comentário: os indivíduos $(\mathrm{n}=2)$ observados assemelham-se quanto ao contorno valvar, padrão de estrias e dimensões aos espécimes encontrados por Metzeltin \& Lange-Bertalot (1998, plate 157, figs 6-9) no Lago Calado e nas Guyanas (Rio Essequibo e Demerara). Esta espécie é primeira citação para o estado do Pará.

7. Gomphonema pantropicum Reichardt in Fottea, Olomuc, 15(1): 27-38, figs. 87-107, 2015.

Fig. 4a,b

Valvas clavado-lanceoladas, polo apical ligeiramente capitado alargado- arredondado, polo basal atenuado arredondado, valvas menores com polo apical capitado. Área axial alargando-se em direção à área central. Rafe linear com extremidades proximais retas. Estrias radiadas, algumas vezes paralelas na região central. Estigma presente no lado oposto da estria mediana encurtada. Comprimento 30,8-53,5 $\mu \mathrm{m}$, largura 5,6-7, $8 \mu \mathrm{m}$, estrias 8-12 em $10 \mu \mathrm{m}$ na área central, $11-13 \mathrm{em} 10 \mu \mathrm{m}$ nos polos. Material examinado: HSTM 000049, HSTM 000054, HSTM 000055, HSTM 000056, HSTM 000057, HSTM 000058 , HSTM 000059.

Comentário: a população $(n=41)$ encontrada no material examinado assemelha-se ao material tipo (descrito originalmente como Gomphonema subtile var. malayensis) registrado por Hustedt (1942) em lagos das Ilhas de Leyte e Mindanao no arquipélago Malaio. Os indivíduos menores apresentaram polos apicais menos capitados semelhante ao observado no material tipo. Gomphonema subtile var. rotundata A. Cleve (Cleve \& Euler 1955: 177, fig. 1268 c) difere de G. pantropicum, por apresentar menor largura, além disso foi registrada somente em material fóssil encontrado na Finlândia. Gomphonema subtile var. sagitta (Schumann) Cleve outra espécie semelhante apresenta forma cuneada no polo apical.

8. Gomphonema parvulum var. lagenula (Kützing) Frenguelli in Bol. Acad. Nac. Ciênc. Córd., v. 27, p.68, pl. 6, fig.16, 1923 . Figs. 4c,d; 3i-k Valvas lanceoladas, polo apical subcapitado, polo basal subcapitado. Área axial estreita, linear. Rafe levemente sinuosa com extremidades proximais retas. Estrias paralelas a levemente radiadas. Estigma presente no lado oposto da estria mediana encurtada. Em MEV, vista externa, observa-se aréolas arredondas (Fig. 3i). Presença de campo de poros no polo basal, vista externa (Fig. $3 \mathrm{k}$ ). Aréolas arredondadas a retangulares (4 aréolas em $1 \mu \mathrm{m}$ ) ocluídas por vola (Fig. 3j). Comprimento $16,7-29,1 \mu \mathrm{m}$, largura 5,2-8 $\mu \mathrm{m}$, estrias $13-20 \mathrm{em}$ $10 \mu \mathrm{m}$ na área central e 15-16 estrias nos polos.

Material examinado: HSTM 000054, HSTM 000055 , HSTM 000057, HSTM 000058, HSTM 000059.

Comentário: a população $(n=46)$ baseou-se no material observado e ilustrado por Frenguelli (1923, plate.6, fig. 16). Esta espécie foi encontrada na região Amazônica, sendo citada por Metzeltin \& Lange-Bertalot (1998, plate 151, figs 12-17) como Gomphonema lagenula Kützing assemelhando-se morfologicamente aos espécimes estudados. O material registrado no presente estudo encontrase dentro dos limites de comprimento e largura (compr. 11-30 $\mu \mathrm{m}$, larg. 5,3-8 $\mu \mathrm{m}$ ) apresentados por Wetzel (2011). A variação de medidas (compr. 24-38 $\mu \mathrm{m}$ ) citada por Metzeltin \& Lange-Bertalot (1998) e por Frenguelli (compr. 30-32 $\mu \mathrm{m}$ ) deve-se, 

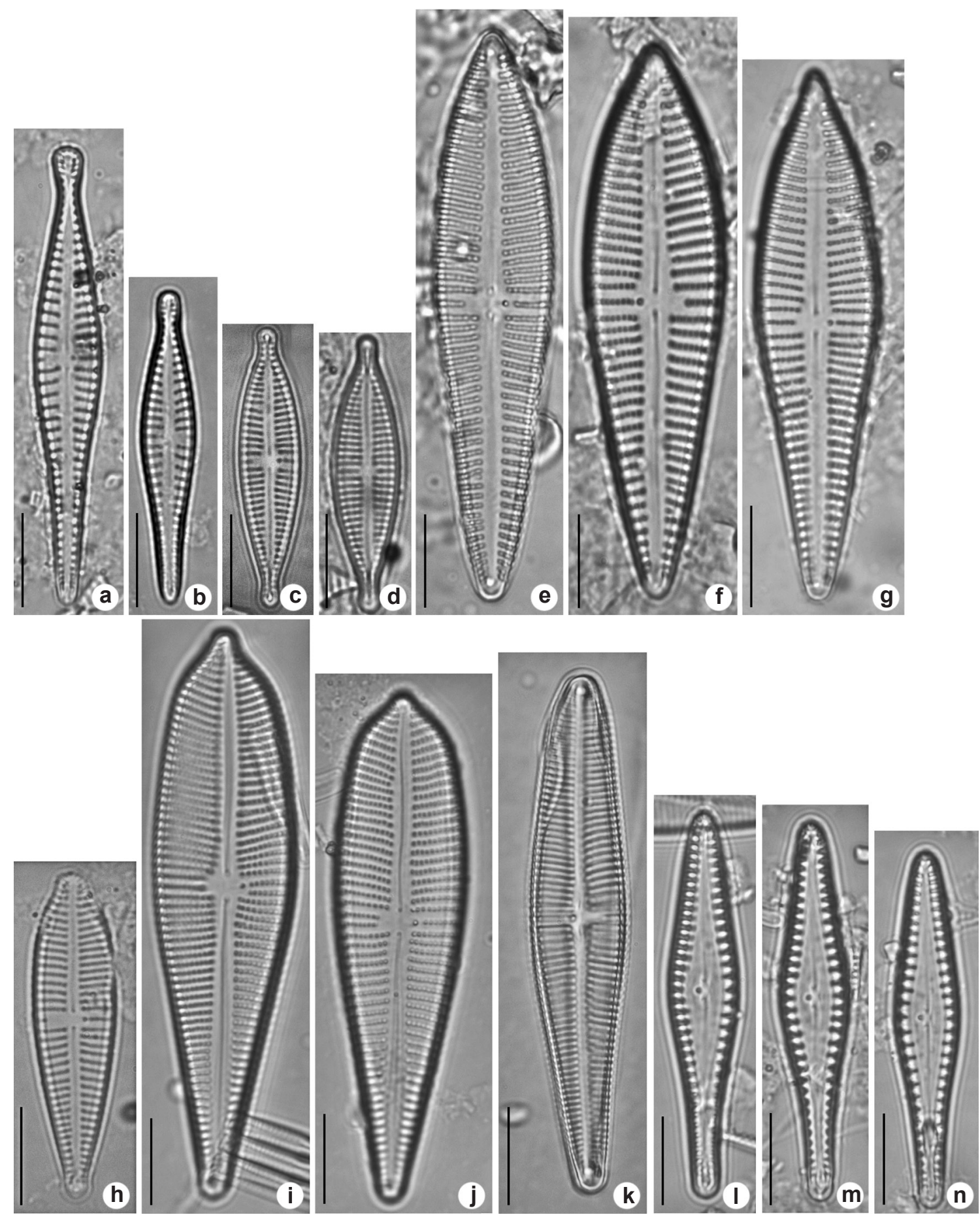

Figura 4 - Imagens em microscopia óptica - a,b. G. pantropicum; c,d. G. parvulum var. lagenula; e-g. G. perapicatum; h. G. pseudoaugur; i,j. G. turris; k. Gomphonema sp. 1; 1-n. Gomphonema sp. 2. Escala $=10 \mu \mathrm{m}$.

Figure 4 - Images in light microscopy - a,b. G. pantropicum; c,d. G. parvulum var. lagenula; e-g. G. perapicatum; h. G. pseudoaugur; i,j. G. turris; k. Gomphonema sp. 1; 1-n. Gomphonema sp. 2. Scale $=10 \mu \mathrm{m}$. 
provavelmente, pela observação de menor número de indivíduos do que por nós observados.

9. Gomphonema perapicatum Metzeltin \& LangeBertalot in Lange-Bertalot. Icon. Diatom., v.18, p. 150, pl. 210, figs. 6-10. $2007 . \quad$ Figs. 31,m; 4e-g

Valvas clavado-lanceoladas, polo apical cuneado, polo basal atenuado arredondado. Área axial alargando-se em direção a área central. Rafe levemente sinuosa com extremidades proximais retas. Estrias levemente radiadas na região central da valva, mais distantes entre si na área central e radiadas próximo aos polos. Estigma presente no lado oposto da estria mediana encurtada. Em $\mathrm{MEV}$, vista interna, o estigma em forma de fenda transversal situado no lado oposto da estria central mais curta, e as extremidades proximais da rafe são levemente fletidas para o lado do estigma (Fig. $3 \mathrm{~m}$ ). Comprimento 58,1-66,2 $\mu \mathrm{m}$, largura 12,9-16,5 $\mu \mathrm{m}$, estrias 7-9 em $10 \mu \mathrm{m}$ na área central, 8-11 estrias em $10 \mu \mathrm{m}$ nos polos.

Material examinado: HSTM 000054, HSTM 000055, HSTM 000059.

Comentário: os indivíduos $(\mathrm{n}=3)$ observados assemelham-se aos espécimes apresentados por Metzeltin \& Lange-Bertalot (2007) encontrados na Guyana, América do Sul, com medidas (compr. 40$75 \mu \mathrm{m}$ larg. 12-13,5 $\mu \mathrm{m}$ ). No material identificado foi possível observar um indivíduo com menor largura, enquanto que as demais medidas conferem com o material tipo. Gomphonema perapicatum diferencia-se de Gomphonema apicatum Ehrenberg pelo formato do polo apical (acuminado-apiculado) e pela maior largura que esta espécie apresenta (Patrick \& Reimer 1975). Esta espécie é primeira citação para o estado do Pará.

10. Gomphonema pseudoaugur Lange-Bertalot in Archiv für Hydrob. Suppl. p. 184-219.1979.

Fig. $4 \mathrm{~h}$

Valvas lanceoladas, polo apical sub-rostrado, polo basal atenuado arredondado. Área axial estreita, linear. Rafe linear com extremidades proximais retas. Estrias paralelas a levemente radiadas. Estigma presente no lado oposto da estria mediana encurtada. Aréolas arredondadas. Comprimento $32,8 \mu \mathrm{m}$, largura $8,5 \mu \mathrm{m}$, estrias $12 \mathrm{em} 10 \mu \mathrm{m}$ na área central e 14 estrias em $10 \mu \mathrm{m}$ nos polos.

Material examinado: HSTM 000057.

Comentário: o indivíduo $(\mathrm{n}=1)$ observado assemelha-se morfologicamente aos espécimes descritos por Krammer \& Lange-Bertalot (1986, plate 159, figs.1-4) e conferindo quanto às dimensões (compr. 25-55 $\mu \mathrm{m}$ larg.7-10 $\mu \mathrm{m}$ e estrias 9-12 em $10 \mu \mathrm{m})$. Esta espécie foi observada por Wetzel (2011) no Rio Negro apresentando maiores dimensões (compr. 75-108 $\mu \mathrm{m}$, larg. 18-24 $\mu \mathrm{m}$ e estrias $6 \mathrm{em} 10 \mu \mathrm{m})$. Os indivíduos encontrados por Tremarin et al.(2009) no Rio Maurício assemelhamse quanto às dimensões e padrão de estrias (compr. 26,1-41,1 $\mu \mathrm{m}$, larg. 7,9-10 $\mu \mathrm{m}, 3$ e estrias 10-16 em $10 \mu \mathrm{m})$. Esta espécie é primeira citação para o estado do Pará.

11. Gomphonema turris Ehrenberg in Abh. König. Akad. Wiss. Berlin. p.291-445, pl.4, 1843.

Fig. 4i,j

Valvas clavadas, polo apical cuneadosubrostrado, cuneado-rostrado a cuneado-obtuso, polo basal atenuado arredondado. Área axial linear. Rafe levemente sinuosa com extremidades proximais retas. Estrias levemente radiadas e pontuadas. Estigma presente, junto à estria mediana encurtada. Comprimento 62-69,2 $\mu \mathrm{m}$, largura $15,7-16 \mu \mathrm{m}$, estrias 9-12 em $10 \mu \mathrm{m}$ na área central, 10-11 estrias em $10 \mu \mathrm{m}$ nos polos.

Material examinado: HSTM 000051.

Comentários: os indivíduos $(\mathrm{n}=3)$ conferem com o material registrado em Manaus, Amazonas e ilustrado por Lange-Bertalot, (1995, plate. 37, figs 5, 6). Este táxon assemelha-se à Gomphonema turris Ehrenberg. f. coarctata Frenguelli encontrado na Argentina por Sar (2009, p. 177, figs. 35-36) diferindo, no entanto, nas dimensões e densidade de estrias (compr. $75-108 \mu \mathrm{m}$, larg. 18-24 $\mu \mathrm{m}$ e estrias $6 \mathrm{em} 10 \mu \mathrm{m})$.

\section{Gomphonema sp. 1}

Fig. $4 \mathrm{k}$

Valvas lanceoladas, polo apical arredondado e alargado semelhante ao polo basal. Área axial estreita, linear. Rafe linear com extremidades proximais retas. Estrias paralelas levemente radiadas. Estigma presente no lado oposto da estria mediana encurtada. Comprimento $65,2 \mu \mathrm{m}$, largura $12 \mu \mathrm{m}$, estrias $11-12 \mathrm{em} 10 \mu \mathrm{m}$ na área central e 14 estrias em $10 \mu \mathrm{m}$ nos polos.

Material examinado: HSTM 000051.

Comentário: $\mathrm{O}$ indivíduo observado $(\mathrm{n}=1)$ assemelha-se morfologicamente a Gomphonema candelariae Frenguelli (Sar et al. 2009, pl. 9, fig. 17) mas, difere pelas dimensões (compr. 80-100 $\mu \mathrm{m}$ larg. $15-17 \mu \mathrm{m}$ e estrias 5-6 em $10 \mu \mathrm{m})$.

13. Gomphonema sp. 2

Fig. 41-n

Valvas rombo-lanceoladas, polo apical arredondado, polo basal relativamente mais 
estreito que o apical. Área axial alargando-se em direção à área central. Rafe linear com as extremidades proximais levemente fletidas para o lado do estigma. Estrias curtas, grosseiras paralelas levemente radiadas nos ápices. Estigma próximo às extremidades proximais da rafe. Aréolas alongadas. Comprimento $38,8-52,3 \mu \mathrm{m}$, largura 7,7-8,5 $\mu \mathrm{m}$, estrias 7-9 em $10 \mu \mathrm{m}$ na área central, 10-11 estrias em $10 \mu \mathrm{m}$ nos polos.

Material examinado: HSTM 000048.

Comentário: os indivíduos $(\mathrm{n}=7)$ assemelhamse morfologicamente e morfometricamente aos espécimes de Gomphonema lepidum Fricke apresentados por Metzeltin \& Lange-Bertalot (1998, pl.152, figs. 6-12) registrados no rio Tapajós, Santarém-PA. No entanto, ao analisarmos o material tipo descrito por Fricke in Schmidt (1904, pl. 248, fig. 15), observou-se que o iconotipo de G. lepidum possui valva clavada, ápices agudos, área axial mais estreita e estrias mais alongadas transapicalmente diferindo dos indivíduos encontrados no presente estudo e também do material apresentado por Metzeltin \& Lange-Bertalot (1998).

\section{Agradecimentos}

À Coordenação de Aperfeiçoamento de Pessoal de Nível Superior (CAPES), a concessão de bolsa de Mestrado à primeira autora e de pós-doutorado da segunda autora. Ao Conselho Nacional de Desenvolvimento Científico e Tecnológico $(\mathrm{CNPq})$, a bolsa de Pesquisa à terceira autora. Ao Programa de Pós-graduação em Recursos Aquáticos Continentais Amazônicos (PPG-RACAM-Ufopa) e ao Programa de Pósgraduação Doutorado Sociedade, Natureza e Desenvolvimento (Ufopa). À Universidade Federal do Oeste do Pará (Ufopa), ao Museu de Ciências Naturais da Fundação Zoobotânica do Rio Grande do Sul (MCN-FZB/RS), ao Centro de Microscopia e Microanálise (CMM-UFRGS), ao Sr. Mário R. Pedreiro e à Eletronorte, o apoio logístico.

\section{Referências}

Barber HG \& Haworth EY (1981) A guide to the morphology of the diatom frustule. The Freshwater Biological Association 44: 1-112.

Bastos ASM, Costa VB, Costa DD, Martins RCVS, Paiva RS \& Camargo-Zorro M (2011) Abundância e frequência de ocorrência de algas epilíticas das das localidades da Boa Esperança e Arroz Cru do setor do médio rio Xingu/PA-Brasil. Boletim Técnico Científico do Cepnor 11: 59-70.

Bes D, Ector L,Torgan LC \& Lobo EA (2012) Composition of the epilithic diatom flora from a subtropical river, Southern Brazil. Iheringia 67: 93-125.

Bicudo CEM \& Menezes M (2006) Gêneros de algas de águas continentais do Brasil. $2^{a}$ edição. Rima, São Carlos. 502p.

Bittencourt-Oliveira MC (1993a) Ficoflórula do Reservatório de Balbina, estado do Amazonas, III: Classe Zygnemaphyceae. Revista Brasileira de Biologia 53: 477-488.

Bittencourt-Oliveira MC (1993b) Ficoflórula do Reservatório de Balbina, estado do Amazonas, II: Ulothricales e Volvocales (Chlorophyceae) e Classe Oedogoniophyceae. Revista Brasileira de Biologia 53: 469-475.

Bittencourt-Oliveira MC (1993c) Ficoflórula do Reservatório de Balbina, estado do Amazonas, I: Chlorococcales (Chlorophyceae). Revista Brasileira de Biologia 53: 113-129.

Bittencourt-Oliveira MC (1994) Ficoflórula do Reservatório de Balbina, estado do Amazonas, Brasil: Nostocophyceae, Cryptophyceae e Tribophyceae. Hoehnea 21: 163-173.

Bittencourt-Oliveira MC (1995) Ficoflórula do Reservatório de Balbina, estado do Amazonas, Brasil: Bacillariophyceae e Chrysophyceae. Hoehnea 22: 147-157.

Bittencourt-Oliveira MC (1997) Euglenophyceae do Reservatório de Balbina, estado do Amazonas. Hoehnea 24: 21-35.

Burliga AL, Torgan LC \& Beaumord AC (2007) Eunotia ariengae sp. nov., an epilithic diatom from Brazilian Amazon. Diatom Research 22: 247-253.

Burliga AL \& Kociolek JP (2010) Kobayasiella species from Carajás National forest, Amazonia, Brazil. Diatom Research 25: 235-250.

Burliga AL \& Kociolek JP (2012) Four new Eunotia Ehrenberg species (Bacillariophyceae) from pristine regions of Carajás National Forest, Amazonia, Brazil. Nova Hedwigia 141: 53-70.

Castro JGD (1999) Composição da Comunidade de diatomáceas perifíticas do rio Jaú, Amazonas, Brasil. Acta Amazônica 4: 583-606.

Cleve PT \& Euler A (1955) Die Diatomeen von Sschweden und Finnland. Kungliga Svenska Vetenskapsakadememiens Handligar. FjärdeSerien 4: $1-158$.

Diaz-Castro JJ, Souza-Mosimann RM, Laudares-Silva R \& Forsberg BR (2003) Composition of the periphytic diatom community of the Jaú river, Amazonas, Brazil. Acta Amazonica 33: 583- 606.

Ehrenberg CG (1843) Verbreitung und Einflufs des mikroskopischen Lebens in Süd-und Nord-Amerika. Abhandlungen der Königlichen Akademie der Wissenschaften zu Berlin. Pp.291-445.

Faria DM, Tremarin PI \& Ludwig TAV (2010) Diatomáceas perifíticas da represa Itaqui, São José dos Pinhais, Paraná: Fragilariales, Eunotiales, 
Achnanthales e Gomphonema Ehrenberg. Biota Neotropica 10: 415-426.

França RCS, Lopes MRM \& Ferragut C (2011) Structural and successional variability of periphytic algal community in a Amazonian lake during the dry and rainy season (Rio Branco, Acre). Acta Amazonica 41: 257-266.

Frenguelli J (1923) Contribuciones para la sinopses de las Diatomeas Argentinas. Boletín de la Academia Nacional de Ciências em Córdoba 27: 13-119.

Hendey N (1964) An introductory account of the smaller algae of British waters - V. Baciilariophyceae (Diatoms). Fishery Investigations Series IV. Her Majesty's Stationery Office, London. 317p.

Hustedt F (1930) Bacillariophyta (Diatomeae) In: Pascher A(ed.) Die Süsswasser-Flora Mitteleuropas. Vol. 10. Gustav Fischer, Jena. Zweite Auflage. 466p.

Hustedt F (1942) Süßwasser-Diatomeen des indomalayischen Archipels und der Hawaii-Inseln. Internationale Revue der gesamten Hydrobiologie und Hydrographie 42: 1- 252.

Junk WJ, Robertson BA, Darwich AJ \& Vieira I (1981) Investigações limnológicas e ictiológicas em CuruáUna, a primeira represa hidroelétrica na Amazônia Central. Acta Amazonica 11: 689-716.

Krammer K \& Lange-Bertalot H (1985) Naviculaceae Neue und wenig bekannte Taxa, neue Kombinationen und Synonyme sowie Bemerkungen zu einigen Gattungen. Bibliotheca Diatomologica 9: 5-230.

Krammer K \& Lange-BertalotH(1986) Bacillariophyceae. Naviculaceae. In: Ettl H, Gerloff L, Heynig H \& Mollenhauer D (eds.) Süsswasser flora von Mittleleuropa. Vol. 2. Gustav Fischer Verlag, Sttuttgart. 876p.

Krammer K \& Lange-BertalotH(1991) Bacillariophyceae. Achnanthaceae Kritische Erganzingen zu Navicula (Lineolatae) und Gomphonema. In: Ettl et al. (eds.) Susswasserflora von Mitteleuropa. Vol. 2/4. Gustav Fisher Verlag, Stuttgart. 436p.

Krasske G (1948) Diatomeen Tropischer Moorasen. Svensk Botanisk Tidskrift 42: 404-403.

Kociolek JP (2016) Gomphonemoids. Special Workshops. In: Pienitz R \& Zinmermann C (eds.) $24^{\text {th }}$ International Diatom Symposium, Québec, Canadá. Université Laval, Québec. 271p.

Lange-Bertalot H (1979) Toleranzgrenzen und Populations dynamik bentischer Diatomeen bei unterschiedlich starker Abwasserbelastung Archiv für Hydrobiologie, Supplement 56: 184-219.

Lange-Bertalot H (1993) 85 neue Taxa und über 100 weitere neu definierte Taxa ergänzend zur Süßwasserflora von Mitteleuropa. Bibliotheca Diatomologica 27: 1-164.

Lange-Bertalot H (1995) Die Diatomeen (Bacillariophyceae) in Ehrenberg's material von Cayenne, Guyana Gallica (1843). In: Lange-Bertalot
H (ed.) Iconographia diatomologica. Annotated diatom micrographs. Vol. 1. Taxonomy. Koeltz Scientific Books, Königstein. Pp. 7-99.

Metzeltin D \& Lange-Bertalot H (1998) Tropical diatoms of South America I: about 700 predominantly rarely known or new taxa representative of the neotropical flora. In: Lange-Bertalot H (ed.) Iconographia diatomologica. Vol. 5. Koeltz Scientific Books, Königstein. 695p.

Metzeltin D \& Lange-Bertalot H (1998) Tropical diatoms of South America II: about 700 predominantly rarely known or new taxa representative of the neotropical flora. In: Lange-Bertalot $\mathrm{H}$ (ed.) Iconographia diatomologica. Vol. 18. Koeltz Scientific Books, Königstein. 877p.

Metzeltin D, Lange-Bertalot H \& García- Rodríguez F (2005) Diatoms of Uruguay. Compared with other taxa from South America and elsewhere. In: LangeBertalot H (ed.) Iconographia diatomologica. Vol. 15. ARG Gantner Verlag, KG, Ruggell. 736p.

Metzeltin D \& Lange-Bertalot H (2007) Tropical diatoms of South America II. Special remarks on biogeography disjunction. In: Lange-Bertalot $\mathrm{H}$ (ed.) Iconographia diatomologica. Vol. 18. ARG Gantner Verlag KG, Ruggell. 877p.

Patrick R \& Reimer CW (1975) The diatoms of the United States exclusive of Alaska and Hawaii. Entomoneidaceae, Cymbellaceae, Gomphonemaceae, Epithemiaceae. Vol. 2. Academy of Natural Sciences of Philadelphia, Monographs. $213 p$.

Putz R \& Junk WJ (1997) Phytoplankton and Periphyton. In: Junk WJ (ed.) The central Amazon floodplain: ecology of a pulsing system. Ecological Studies 126. Springer, London. Pp. 207-222.

Reichardt E (2015a) Gomphonema gracile Ehrenberg sensu stricto et sensu auct. (Bacillariophyceae): a taxonomic revision. Nova Hedwigia 101: 367-393.

Reichardt E (2015b) Taxonomy and distribution of Gomphonema subtile Ehrenberg (Bacillariophyceae) and six related taxa. Fottea. Olomouc 15:27-38.

Round FE, Crawford RM \& Mann DG (1990) The diatoms, biology \& morphology of the genera. Cambridge University Press, Cambridge. 741p.

Sar EA, Sala SE, Sunesen I, Henninger MS \& Montastruc M (2009) Catálogo de los géneros, especies y taxa infraespecíficos erigidos por J. Frenguelli (Catalogue of the genera, species and infraspecific taxa erected by J. Frenguelli). Diatom Monographs 10: 1-419.

Schmidt A (1904) Atlas der Diatomaceen-kunde. Series VI (Heft 62-63). O.R. Reisland, Leipzig. Pp. 245252.

Schneck F, Torgan LC \& Schwarzbold A (2008) Diatomáceas epilíticas em riachos de altitude no sul do Brasil. Rodriguésia 59: 325-338. 
Smith W (1856) A synopsis of British Diatomaceae. Vol. 2. John Van Voorst, London. 239p.

Stosch HAV (1970) Methoden zur preparation keleiner oder zarter kieselelemente fur die elektronen und lichtmikroskopie, insbesondere von diatomeen und bei geringen materialmengen. Zeitschrift fur wissenschaftlichen Mikroscopie 70: 29-32

Tremarin PI, Ludwig TAV, Bertolli LM, Faria DM \& Costin JC (2009) Gomphonema Ehrenberg e Gomphosphenia Lange-Bertalot (Bacillariophyceae) do Rio Maurício, Paraná, Brasil. Biota Neotropica 9: 111-130.

Tremarin PI \& Ludwig TV (2008) Anorthoneis dulcis Hein: first record in South America. Diatom Research 23: 213-220

Tremarin PI, Straube A, Tibiriça CE, Castro E, Wojciechowski J, Moreira Filho H \& Ludwig TAV (2015) First record of Capartogramma paradisiaca Novelo, Tavera \& Ibarra (Diatomeae) in South America. Revista Brasileira de Botânica 38: 1-5.
Vieira I (2000) Freqüência, constância, riqueza e similaridade da ictiofauna da bacia do rio CuruáUna, Amazônia. Revista Brasileira de Zoociências 2: 41-50.

Wetzel CE (2011) Biodiversidade e distribuição espacial de diatomáceas (Bacillariophycea) na bacia hidrográfica do Rio Negro, Amazonas, Brasil. Vol. 1-3. Tese de Doutorado. Instituto de Botânica, São Paulo. 1922p.

Wetzel CE, Vijver BV, Cox EJ, Bicudo DC \& Ector L (2012a) Tursiocola podocnemicola sp. nov., a new epizoic freshwater diatom species from the Rio Negro in the Brazilian Amazon Basin. Diatom Research 27: 1-8.

Wetzel CE, Lange-Bertalot H, Morales EA, Bicudo DC, Hoffmann L \& Ector L (2012b) Bicudoa amazonica gen. nov. et sp. nov. (Bacillariophyta) a new freshwater diatom from the Amazon basin with a complete raphe loss in the Eunotioid lineage. Phytotaxa 75: 1-18. 